\title{
Article
}

\section{Insights into the Relationship of the Immune System with \\ Neurodevelopmental and Psychiatric Disorders}

Gottfried, Carmem and Bambini-Junior, Victorio

Available at http://clok.uclan.ac.uk/25920/

Gottfried, Carmem and Bambini-Junior, Victorio ORCID: 0000-0002-8590-6770 (2019) Insights into the Relationship of the Immune System with Neurodevelopmental and Psychiatric Disorders. Neuroimmunomodulation, 25 . pp. 243-245. ISSN 1021-7401

It is advisable to refer to the publisher's version if you intend to cite from the work. http://dx.doi.org/10.1159/000496180

For more information about UCLan's research in this area go to http://www.uclan.ac.uk/researchgroups/ and search for <name of research Group>.

For information about Research generally at UCLan please go to http://www.uclan.ac.uk/research/

All outputs in CLoK are protected by Intellectual Property Rights law, including Copyright law. Copyright, IPR and Moral Rights for the works on this site are retained by the individual authors and/or other copyright owners. Terms and conditions for use of this material are defined in the policies page. 


\section{Insights about the relationship of the immune system with neurodevelopmental and psychiatric disorders.}

Carmem Gottfried ${ }^{1,2,3,4}$, Victorio Bambini-Junior ${ }^{1,2,3,5}$

1. Translational Group in Autism Spectrum Disorder - GETTEA, Clinical Hospital of Porto Alegre, Porto Alegre, Brazil

2. Autism Wellbeing And Research Development (AWARD) Institute, BR-UK.

3. Brazilian National Institute of Science and Technology on neuroimmunomodulation (INCT-NIM), Rio de Janeiro, Brazil

4. Department of Biochemistry, Federal University of Rio Grande do Sul, Porto Alegre, RS, Brazil.

5. School of Pharmacy and Biomedical Sciences, University of Central Lancashire, Preston, UK.

Neurodevelopmental and psychiatric disorders correspond to a range of conditions that affect the developing brain, generating long-term global effects, such as autism spectrum disorder (ASD), schizophrenia and attention deficit hyperactivity disorder (ADHD) [1]. Those are quite heterogeneous conditions that share common features including alterations in the immune system and hormonal modulation of T-cell development [2].

The brain of a child is in continuous development and does not represent a miniature replica of an adult brain. It is constantly undergoing structural and neurochemical changes due to the continuous stimulation provided by the environment in which it develops. In this context, the immune system plays a central role, directly influencing and modulating this highly plastic brain. The impact can be varied, depending on the parental genetic/epigenetic background, and environmental risk factors [3], affecting neurodevelopmental stages during pregnancy, perinatal period or childhood (Figure 1). 
Neuroimmunomodulation is a field of knowledge that is continuously and rapidly advancing, e.g. three decades ago it was considered that the central nervous and immune systems worked independently, with little or no interactions between them. Recently, the discovery of the central nervous system lymphatic vessels [4] suggests that malfunction of the meningeal lymphatic vessels could also be involved in neurological disorders.

Currently, we know that there is a vast interface of communication between the brain-gut axis and microbiota [5], involving changes in brain barriers permeability [6]. The human microbiome comprises trillions of commensal microbes, including bacteria, fungi, and viruses, affecting epigenetic mechanisms, metabolic activity, and immune system function. The alterations in the balance between the communication of homeostatic systems, such as the nervous, endocrine and immune systems, make us more prone to different disorders (Figure 2). Although many studies concerning the neuroimmunoendocrine circuitry have emerged in recent years, the potential pathways involved in the triggering and pathophysiology of psychiatric and neurodevelopmental disorders, as well as the specific role played by neuroimmune axes in this process, still require an extensive investigation. This might, in turn, reward us with new therapeutic targets and preventive strategies for these disorders.

In this special issue we invited distinguished researchers to discuss 'immune subjects' relevant to the pathophysiology and aetiology of neurodevelopmental and psychiatric conditions.

Liberman et al. provide a comprehensive review of the neuroimmune alterations found in several psychiatric disorders in the article 'Neuroimmune 
and inflammatory signals in complex disorders of the CNS'. The main topics are: (1) peripheral immune findings and depression; (2) ASD and the central effects of long-term pro-inflammatory stimuli, including morphological alteration; (3) brain repair and the role to microglia, and; (4) the relation between neurodegenerative disorders and neuroinflammation.

The article 'Intracellular Pathogens Infections and Immune Response in Autism' by Abib et al. focus on the analysis of the clinical findings linking perinatal infections and ASD. The central role of IL-6, which has also been used to induce preclinical models of ASD in rodents and non-human primates, is also investigated.

In the article 'Reduced CD4 T lymphocytes in lymph nodes of the mice model of autism induced by valproic acid', Baronio et al. present findings related to immune alterations in one of the main models for the study of ASD, with a decrease in T cells in the lymph nodes.

Deckmann et al. present a recollection of the immune findings in the animal model of ASD induced by VPA in the article 'Neuroimmune alterations in autism: a translational analysis focusing on the animal model of autism induced by prenatal exposure to valproic acid'. Interestingly, they highlight the direct links between the model with actual findings in humans with ASD, also providing a comprehensive outline on the current literature in the area.

The extensive findings of immune alterations in patients with ASD place the immune system as a potential candidate for therapeutic interventions. In the article 'Immunological Dysfunction in Autism Spectrum Disorder: A Potential Target For Therapy', Marchezan et al. present a comprehensive list of interventions targeting the immune system. They also evaluate and analyse the 
outcomes, providing valuable insights into the pathways that could be further explored and the ones that, unfortunately, are not offering promising results.

Following the recent outbreak of Zika Virus (ZIKV) and its link to congenital malformations, Vianna et al. explore the potential relationship between ZIKV and ASD. The article 'ZIKV as a possible risk factor for ASD:

neuroimmunological aspects' explores the maternal immune alterations and the foetal brain infection induced by ZIKV, and how this could predispose those infected to ASD

Leffa et al. offers an analysis of the evidence linking ADHD to immune alterations in the article 'A review on the role of inflammation in AttentionDeficit/Hyperactivity Disorder'. They stress that, despite the evidence suggesting a strong correlation between ADHD and the immune system, further studies are instrumental to determine a causative link.

In the article 'The relationship between cytokines and verbal memory in individuals with schizophrenia and their unaffected siblings', Rebouças et al. compare the verbal memory of patients with schizophrenia with their unaffected siblings and healthy controls. Their scores are analysed and corelated to the peripheral immune findings, providing important insights about neuroimmune biomarkers in chronic and acute psychotic states.

Therefore, this special issue addresses the relationship between neuroimmunomodulation, developmental and psychiatric conditions, highlighting conceptual pitfalls and puzzling new outstanding questions and directions for further research. We hope this alternative approach to disorders traditionally approached through exclusive neural optics, will be insightful and exciting for you readers. Enjoy! 


\section{Acknowledgements}

Special thanks go to the Editor of Neuroimmunomodulation, Professor Wilson Savino, for the opportunity we have been given in coordinating a special issue on neurodevelopmental and psychiatric disorders. We would also like to thank the online infographic maker, Mind the Graph (attribution share-alike 4.0 licensing) for the templates of schematic figures.

\section{References}

1 DSM-5: Neurodevelopmental Disorders; in : Diagnostic and Statistical Manual of Mental Disorders. American Psychiatric Association, 2013. DOI: 10.1176/appi.books.9780890425596.dsm01

2 Savino W, Mendes-da-Cruz DA, Lepletier A, Dardenne M: Hormonal control of T-cell development in health and disease. Nat Rev Endocrinol 2016;12:77-89.

3 Tzika E, Dreker T, Imhof A: Epigenetics and Metabolism in Health and Disease. Front Genet 2018;9:361.

4 Louveau A, Smirnov I, Keyes TJ, Eccles JD, Rouhani SJ, Peske JD, et al.: Structural and functional features of central nervous system lymphatic vessels. Nature 2015;523:337-341.

5 Cussotto S, Sandhu K V., Dinan TG, Cryan JF: The Neuroendocrinology of the Microbiota-Gut-Brain Axis: A Behavioural Perspective. Front Neuroendocrinol 2018;51:80-101.

6 Liebner S, Dijkhuizen RM, Reiss Y, Plate KH, Agalliu D, Constantin G: Functional morphology of the blood-brain barrier in health and disease. 
Acta Neuropathol 2018;135:311-336. 


\section{Legends of Figures}

Figure 1. Genetic/epigenetic background associated with environmental risk factors impacting life. A. Parental background during fertilization: The parental genetic background, coupled to epigenetic influences and environmental risk factors during pregnancy may contribute to the triggering of neurodevelopmental and psychiatric disorders. The impact of an environmental risk factor on the outcome is also dependent on particular neurodevelopmental windows. B. The influence of genetic and environmental risk factors during embryogenesis: The above-mentioned background can influence embryogenesis by means of a looping of changes with immunological and endocrine responses, which may pass through a pro-inflammatory and prooxidant framework. It is important to point out that the gestational condition undergoes changes in the immunological profile to avoid embryonic rejection. If the embryo is exposed to an environmental risk factor, associated to the particular maternal immune profile, it can have an impact on the neural development, with short, medium- and long-term responses, associated to a neuroimmunoendocrine fingerprint in the offspring. C. Postnatal timeline: Beyond negative influences on embryogenesis timeline, impacts and traumas in the first months of life may undergo epigenetic changes, associated with the neuroimmunoendocrine fingerprint with consequences throughout life.

Figure 2. The developmental crosstalk after embryonic impact through genetic/epigenetic background and environmental risk factor. Growing evidence suggests that immune system activation early in life account for variability in behavioral and psychiatric disorders in an intricate crosstalk 
through life, influencing neuro-endocrine systems associated with the stress response. The gut-brain axis involves bidirectional communication between the central and the enteric nervous systems, interconnected to the microbiome, with neural, endocrine and immune links. Immunological changes may also involve brain barrier permeability, increased blood cell uptake into the brain, with a looping of neuroinflammation and neuroimmune abnormalities common in neurodevelopmental and psychiatric disorders. 